\title{
Activity of single nucleotide polymorphic variants of human AP-endonuclease 1
}

\author{
A.T. Davletgildeeva ${ }^{1,2 *}$, I.V. Alekseeva ${ }^{1}$, O.S. Fedorova ${ }^{1,2}$, N.A. Kuznetsov ${ }^{1,2}$ \\ ${ }^{1}$ Institute of Chemical Biology and Fundamental Medicine SB RAS, Novosibirsk, Russia \\ ${ }^{2}$ Novosibirsk State University, Novosibirsk, Russia \\ *e-mail:davleta94@mail.ru
}

Key words: human APE1, stopped-flow method, pre-steady state kinetics

Motivation and Aim: A single nucleotide replacement in gene can lead to developmental disorders and even to lethal effect if it occurs in reproductive cells or to carcinogenesis if mutation proceeds in somatic cells. At present, five to nine systems of damage correction were distinguished, among which the most active study is conducted with base excision repair (BER) system. This system is polycomponent and functioning due to the coordinated action of more than 10 proteins. One of the major proteins of the BER system is the AP-endonuclease 1 (APE1). The human APE1 is a globular protein of 318 amino acids with a molecular mass $35.4 \mathrm{kDa}$. The main role of APE1 is the correction of apurinic-apyrimidinic (AP) sites generated spontaneously or as a result of $\mathrm{N}$-glycosylase reaction catalyzed by monofunctional DNA-glycosylases. The goal of our research was to analyze the effects of single nucleotide polymorphism of APE1 on the recognition of AP-sites in DNA and catalytic reaction.

Methods and Algorithms: In the current study, we constructed and purified by the standard methods the APE1 SNP variants: Asn222His, Arg237Ala, Arg237Cys, Gly241Arg, Arg274Gln, Pro311Ser, Met270Ala and Met270Thr. These amino acid residues were chosen because some of them are involved in DNA coordination, while others are involved in the catalytic conversion of AP-sites. Comparative kinetic analysis of catalytic processes for APE1 variants was performed by stopped-flow method. The effects of SNP APE1 on conformational changes in enzyme and specific DNA-substrates were analyzed by detection of fluorescence intensities of tryptophan and 2-aminopurine, respectively.

Results: The data obtained in the current work made it possible to determine the kinetic scheme describing interactions of the mutant forms of the enzyme with the DNA substrates, to calculate the rate constants of the elementary stages and to specify the stages of the process affected by these amino acid substitutions. Stopped-flow kinetic study for all mutant forms revealed that such amino acid substitutions change APE1 activity by influence on the different stages of enzymatic process. However, all mutant forms of the enzyme retain the ability to cleave the AP-site.

Conclusion: The natural SNPs in the enzyme APE1 display a significant effect on the recognition, binding and catalytic conversion of AP-sites in DNA.

Acknowledgements: This work was supported by grant from the Russian Foundation for Basic Research (No. 16-04-00037). 